\title{
Fitness cost of resistance: impact on management
}

Book or Report Section

Accepted Version

Mikaberidze, A. and McDonald, B. A. (2015) Fitness cost of resistance: impact on management. In: Ishii, $\mathrm{H}$. and Hollomon, D. W. (eds.) Fungicide Resistance in Plant Pathogens. Principles and a Guide to Practical Management. Springer, pp. 77-89. ISBN 9784431556411 doi: https://doi.org/10.1007/9784-431-55642-8_6 Available at http://centaur.reading.ac.uk/86112/

It is advisable to refer to the publisher's version if you intend to cite from the work. See Guidance on citing.

To link to this article DOI: http://dx.doi.org/10.1007/978-4-431-55642-8_6

Publisher: Springer

All outputs in CentAUR are protected by Intellectual Property Rights law, including copyright law. Copyright and IPR is retained by the creators or other copyright holders. Terms and conditions for use of this material are defined in the End User Agreement. 


\section{www.reading.ac.uk/centaur}

\section{CentAUR}

Central Archive at the University of Reading

Reading's research outputs online 


\title{
Fitness cost of resistance - impact on management
}

\author{
Alexey Mikaberidze \\ Theoretical Biology, Institute of Integrative Biology, ETH Zurich \\ alexey.mikaberidze@env.ethz.ch, tel. +4144632 2602 \\ Bruce A. McDonald \\ Plant Pathology, Institute of Integrative Biology, ETH Zurich \\ bruce.mcdonald@usys.ethz.ch, tel. +4144632 3847
}

Keywords: Fitness, Epidemiology, Host-pathogen interaction, Plant disease, Dynamic diversity

\begin{abstract}
Fungicides are important tools for the management of fungal diseases in many crops. But eventually, most fungicides fail because the treated pathogen population evolves resistance to the fungicide. This chapter focuses on how our knowledge of fitness costs associated with resistance informs strategies of fungicide deployment that help to avoid or delay development of resistance. Many different fungicide deployment strategies should be considered that take into account fungal population genetics as well as the specific agroecosystem. Mono-applications will be replaced by strategies that use several fungicides with different modes of action. Modelling approaches will be needed to inform us regarding the optimum strategies to use under different circumstances. It is clear that fitness costs connected to mutations that encode fungicide resistance will need to be better measured and taken into account in order to design optimum fungicide deployment strategies.

We discuss the importance of fitness costs in assessing the usefulness of fungicide mixtures that contain a high-risk fungicide together with a low-risk fungicide, and the role of population dynamical mathematical models of plant-pathogen interaction. According models, the fitness cost of resistance determines the outcome of competition between the sensitive and resistant pathogen strains. If fitness costs are absent, then the use of the high-risk fungicide in a mixture selects for resistance and the fungicide eventually becomes nonfunctional. If there is a cost of resistance, then an optimal ratio of fungicides in the mixture can be found, at which selection for resistance is expected to vanish and the level of disease control can be optimized.
\end{abstract}

\section{Introduction}

Fungicides are important tools for the management of fungal diseases in many crops. Fungicide applications at the farm and landscape scales keep many important fungal diseases in check, sometimes for several years. But eventually, most fungicides fail because the treated pathogen population evolves resistance to the fungicide. In this chapter, fungicide resistance is treated as a population phenomenon, exhibited mainly at the scale of fields, farms and landscapes, rather than at the level of fungal individuals or cells, even though resistance is usually measured based on growth rates of individual strains. But it should be noted that the emergence of fungicide resistance at the population or landscape level generally begins with mutations that encode resistance at the level of a cell or individual.

Fungicide resistance emerges because pathogens evolve. Evolution requires genetic diversity. Genetic diversity is affected by mutation rate, population size, recombination, gene 
flow, and selection, the same factors that affect an organism's population genetics. Thus the key to developing strategies to avoid the emergence of fungicide resistance lies in understanding the population genetics of fungal pathogens.

The root of the problem of evolution of fungal pathogens is the lack of diversity in agricultural ecosystems (agroecosystems). Since the invention of agriculture 12,000 years ago, crop genetic diversity has declined steadily in agroecosystems globally to facilitate gradual improvements in agricultural production systems, including innovations such as tillage, fertilization, controlled irrigation, and mechanization. The decline in crop genetic diversity at the field scale accelerated rapidly during the last 100 years to increase the efficiency of food production and feed the burgeoning human population. The large-scale mechanization of planting and harvesting operations and the green revolutions that swept through agriculture led to replacement of locally-adapted but genetically diverse land races by genetically uniform but broadly-adapted, high-yielding, dwarf cultivars, further depleting genetic diversity and increasing the environmental homogeneity present in agroecosystems worldwide. The highly mechanized modern industrial agroecosystems now found around the world are extremely productive food factories, but also are highly effective incubators of pathogen evolution (Stukenbrock and McDonald 2008). As a result of the increased planting density and genetic uniformity of host populations in agroecosystems, pathogen population sizes increased, which led to more genetic diversity for selection to act upon by increasing the total number of mutations available at the field scale while simultaneously lowering the effects of genetic drift. Due to these changes in agroecosystems over time, pathogen evolutionary potential likely increased as agricultural pathogens (including fungi, bacteria and viruses) became domesticated and adapted to the agroecosystem environment.

The increasing genetic uniformity of the major crops facilitated the large-scale mechanization of agriculture and simplified all aspects of food production and food processing. The basic trade-off in all agroecosystems is that the increased environmental and genetic uniformity that enables more efficient food production also facilitates pathogen evolution and increases the risk of significant losses due to disease. Thus we are confronted with a dilemma: How can we maximize the many benefits of modern industrial agriculture while minimizing the risks of disease epidemics in these agroecosystems? Is it possible to achieve long-lasting and stable (i.e. durable) disease control, especially if it is based largely on growing resistant cultivars and/or applying fungicides?

Durable disease control will not be achieved unless we increase the overall diversity present in agroecosystems at both the farm and landscape spatial scales. The diversity will need to be dynamic, changing regularly over both time and space, to significantly slow the rate of pathogen adaptation. Fortunately, a wide array of low-technology, medium-technology and high-technology strategies can be used to increase both spatial and temporal diversity in agroecosystems (McDonald 2014). Many of these strategies are well known and oriented around crop husbandry, requiring no additional breeding effort. These crop husbandry strategies include improved crop rotations, species intercropping, and planting smaller fields to increase crop heterogeneity at the farm and landscape scales. Other possibilities include introduction of new crop species into existing agroecosystems, and increasing the overall genetic diversity present in existing crop species by planting mixtures of host cultivars (Mikaberidze et al. 2014a; Mundt 2002) or introducing new genes from their wild relatives.

In this chapter we focus on another aspect of this diversity, namely the chemical diversity represented by the applied fungicides. Static, long-term use of any fungicide alone will impose strong directional selection on the corresponding fungal population that will favor evolved pathogen strains that are resistant to the fungicide. The development of resistance to systemic fungicides that target a single molecule used alone has been widely documented for many decades in all major crops (Brent and Hollomon 2007). Fungicides that inhibit more basic biological processes (e.g. based on copper or thiol groups) used alone are also likely to 
select for less sensitive populations over time as a result of pathogen evolution, though the "erosion" of the activity of these fungicides may be more difficult to document. Strategies of disease control that use more than one fungicide, such as mixtures and alternations of fungicides, have been proposed to delay the emergence of fungicide resistance. But even these mixed strategies are likely to fail after long-term use. We argue that control strategies based on dynamic turnover of fungicide mixtures, i.e. mixtures with components changing over time and space, would be advantageous for delaying the emergence of fungicide resistance in the longer run. In order to predict the effectiveness and durability of fungicide control strategies and optimize the parameters associated with these strategies, i.e. composition, overall dose, proportions of components and the rate of turnover, one needs to use biologically relevant and carefully parameterized mathematical models accompanied by extensive field experimentation. One of the key issues here is to characterize pathogen fitness in these different chemical environments. In this respect the concept of fitness costs becomes useful.

\section{What are fitness costs and how to measure them?}

In some cases fungicide resistance can be conferred by a single point mutation (for example, QoI resistance (Torriani et al. 2009)) that gives mutants full protection from the fungicide. In other cases, several mutations need to be accumulated in the same gene or in different genes in order to gain considerable resistance (Cools 2008; Zhan et al. 2006).

Any mutation that confers fungicide resistance may also disrupt or lower the efficiency of important physiological and biochemical processes (Anderson 2005). Consequently, a resistance mutation may result in lower pathogen fitness. We define the fitness cost of a mutation conferring fungicide resistance, $\rho_{\mathrm{r}}$, as the difference in fitness between the resistant pathogen strain carrying the resistance mutation and the sensitive pathogen strain in the absence of the fungicide. Thus, in order to determine fitness costs, one needs to measure fitness of both sensitive and resistant pathogen strains in the same, nonselective environment.

The overall fitness of fungal plant pathogens can be comprised of several components that correspond to different stages of the pathogen life cycle. These include, but are not limited to, spore production, spore dispersal, infection efficiency, mycelial growth, and the ability to survive between seasons. The ultimate measure of fitness is based on the ability to compete with other strains in a field environment characterized by fluctuating environmental conditions and interactions with a wide variety of host genotypes and competing microbiota. The strains with the highest reproductive fitness will contribute the most genes to future generations. A mark-release-recapture experimental design has been shown to work well to measure competitive fitness for three different cereal pathogens (Zhan and Mcdonald 2013) though these field experiments were not testing fitness costs associated with fungicide resistance. Similar experimental evolution designs should provide useful insight into fitness costs assocated with resistance mutations, though as with all fieldwork, these experiments remain quite costly in terms of resources and labor. Experimental evolution in chemostats, flasks and Petri dishes can also be used to infer fitness costs (Anderson 2005), is much less resource-intensive, and can be combined with next-generation sequence analyses of entire genomes to identify both primary mutations and compensatory mutations associated with resistance. More traditional methods cited below involve comparing growth rates and spore production of resistant and susceptible strains on Petri plates or in planta to infer fitness costs.

When perennial ryegrass (Lolium perenne L.) was inoculated with mixtures of azoxystrobin-resistant and azoxystrobin-sensitive strains of Magnaporthe oryzae, sensitive strains produced more conidia and increased in frequency over time in the absence of the fungicide, consistent with a fitness penalty for azoxystrobin resistance (Ma and Uddin 2009). 
Similarly, the frequency of Cercospora beticola isolates resistant to DMI fungicides slightly but significantly decreased during an epidemic in competition with DMI sensitive strains (Karaoglanidis et al. 2001). In contrast, resistant strains were as fit as sensitive strains of Phytophthora erythroseptica and even displayed a competitive advantage when mefenoxam was absent, suggesting no fitness penalty (Chapara et al. 2011). Similarly, no fitness costs were found in Alternaria alternata resistant to QoI fungicides (Karaoglanidis et al. 2011) or for Phytophthora nicotianae strains resistant to mefenoxam (Hu et al. 2008).

Some studies inferred substantial fitness costs from field monitoring that enabled measurements of changes in frequency of resistant strains over time (for example (Suzuki et al. 2010 and references in Peever and Milgrom 1995). But these findings could result from other factors, including immigration of sensitive isolates, selection for other traits linked to resistance mutations or genetic drift (Billard et al. 2012). Though relatively few carefully controlled experiments have been conducted, the majority indicate that fitness costs associated with fungicide resistance are either low (for example Kim and Xiao 2011: Billard et al. 2012) or absent (for example Corio-Costet et al. 2010; Peever and Milgroom 1994). But in some cases fitness costs were found to be substantial (for example Webber 1988; Kadish and Cohen 1992; Holmes and Eckert 1995; Karaoglanidis et al. 2001; Iacomi-Vasilescu et al. 2008) both in laboratory measurements and in field experiments. Although measurements of fitness costs of resistant mutants performed under laboratory conditions can be informative (for example, Billard et al. 2012) they do not necessarily reflect the costs connected with resistant mutants selected in the field. This is because field mutants are more likely to possess compensatory mutations improving pathogen fitness (Peever and Milgroom 1995) in the field environment. Moreover, a laboratory setting rarely reflects the balance of environmental and host conditions found throughout the pathogen life cycle, since the field environment is much more complex and fluctuates constantly.

A highly relevant measure of pathogen fitness in the context of anti-resistance management strategies is the growth rate of the pathogen population, $r$, at the very start of an epidemic. Figure 1 illustrates the effect of fitness cost on selection for fungicide resistance. The growth rate, $r$, of the sensitive strain decreases as a function of the fungicide dose, $C$ (solid curves).

When resistance is full (left panel), the growth rate of the resistant strain is not affected by the fungicide. Hence, $\mathrm{r}$ remains constant versus $C$ with the magnitude that depends on the fitness cost $\theta$ (dashed lines). In the absence of a fitness cost (upper dashed line in Fig. 1a), resistant mutants have a selective advantage over the sensitive strain as soon as the fungicide is added (i. e. at any $C>0$ ). When there is a fitness cost, the sensitive strain is favored by selection at small doses (cf. the range of doses, where the solid curve is above the lower dashed curves in Fig. 1(a)). But when the dose exceeds a certain threshold value $C_{\mathrm{c}}$, the resistant strain becomes fitter than the sensitive strain. The threshold dose $C_{\mathrm{c}}$ depends on the magnitude of the fitness cost and the dose-response parameters of the sensitive strain that determine how fast its fitness declines with the dose.

In the case of partial resistance (Fig. 1(b)) the fitness of resistant mutants does not remain constant, but declines with the dose $C$. However, it declines slower than the fitness of the sensitive strain. As a result, the range of doses over which the sensitive strain has a selective advantage is expanded as compared to the case of full resistance. Here, the threshold dose $C_{\mathrm{c}}$ depends not only on the magnitude of the fitness cost and the dose-response parameters of the sensitive strain, but also on the dose-response parameters of the resistant strain.

How does an understanding of fitness costs affect anti-resistance management? If there is no fitness cost associated with a resistance mutation, then the pathogen strain carrying this mutation will persist in fungal populations even in the absence of a fungicide. As a result, the corresponding fungicide may permanently lose its effectiveness and there will be no 
benefit associated with using this fungicide in a mixture or alternation. But when fitness costs are present, there are two main conclusions relevant for anti-resistance management. First, selection for resistance can be reversed during the time when the fungicide is absent. However, the rate of reversion is expected to be much slower than the rate of selection for resistance. Also, compensatory mutations that may accompany resistance mutations could make reversion difficult or impossible.

The second conclusion is that low fungicide doses can be applied without selecting for fungicide resistance as long as fitness costs are present. This has a promising application for developing fungicide mixtures that would avoid selection for resistance, but at the same time achieve a desired degree of disease control. We elaborate this idea in the next section with the help of a population dynamical mathematical model that is briefly described in Appendix A.

\section{The role of fitness costs in selection for resistance: insights from mathematical modelling}

The ranges of fungicide dose and cost of resistance at which the sensitive (white) or resistant (grey) pathogen strain is favored by selection are shown in Fig. 2. In all scenarios competitive exclusion is observed: one of the strains takes over the whole pathogen population and the other one is eliminated. If a low-risk fungicide is applied alone, the sensitive strain has a selective advantage across the whole parameter range. When only a high-risk fungicide is applied (Fig. 2(a)), the resistant strain dominates if the fitness cost is lower than the maximum effect of the fungicide $\rho_{\mathrm{r}}<k_{\mathrm{k}}$ and at a fungicide dose higher than a threshold value which increases with the fitness cost (solid curve in Fig. 2(a)). If the fitness cost exceeds $k_{\mathrm{k}}$ (dotted line in Fig. 2(a)), then the sensitive strain dominates at any fungicide dose. Fig. 2(b) shows the outcome when the two fungicides are mixed at equal doses. Here the fitness cost at which the sensitive strain dominates is reduced (vertical dotted line is shifted to the left). As expected, without a fitness cost $\left(\rho_{\mathrm{r}}=0\right)$ the resistant strain becomes favored by selection and will eventually dominate the population whenever the high-risk fungicide is applied, alone or in combination with the low-risk fungicide ( Fig. 2(a,b)).

It is highly desirable to keep existing fungicides effective for as long as possible. From this point of view, an optimal mixture contains the largest proportion of the high-risk fungicide, at which (i) the resistant pathogen strain is not selected and (ii) an adequate level of disease control is achieved. In order to fulfill both of these objectives, the fitness cost of resistance needs to be larger than a threshold value The threshold $\rho_{\mathrm{rb}}$ is shown by the dotted vertical line in Fig. 2(b).

The threshold $\rho_{\mathrm{rb}}$ depends on the proportion of fungicides in the mixture. Adding more of the low-risk fungicide, while keeping the same total dose $C$, reduces the threshold. This diminishes the range of the values for fitness cost over which the resistant strain dominates. On the other hand, adding less of the low-risk fungicide, while again keeping $C$ the same, increases the threshold, which increases the parameter range over which the resistant strain is favored.

Therefore, at a given fitness cost $\rho_{\mathrm{r}}$, one can adjust the fungicide ratio $r_{\mathrm{B}}$ such that $\rho_{\mathrm{r}}>\rho_{\mathrm{rb}}$. This is shown in Fig. 3: the curve shows the critical proportion of the low-risk fungicide $r_{\mathrm{Bc}}$, above which no selection for resistance occurs at any total fungicide dose $C$. One can see from Fig. 3 that if the resistance cost is absent $\left(\rho_{\mathrm{r}}=0\right)$, then the high-risk fungicide should not be added at all if one wants to prevent selection for resistance. At larger fitness costs, the value of $r_{\mathrm{Bc}}$ decreases, giving the possibility to use a larger proportion of the high-risk fungicide without selecting for resistance. Finding an optimum proportion of 
fungicides requires knowledge of both the fitness cost $\rho_{\mathrm{r}}$ and the maximum effect of the fungicide $k_{\mathrm{k}}$.

\section{How can knowledge of fitness costs inform resistance management strategies?}

In the following sections we discuss how the information about fitness costs combined with the modeling insights from the previous section can help us in determining effective strategies for managing fungicide resistance.

\subsection{When are fungicide mixtures effective as a resistance management strategy?}

In cases when fungicide resistance confers no fitness cost (for example Karaoglanidis and Luo 2011), application of a mixture of high-risk and low-risk fungicides will select for resistance. Consequently, the resistant strain will eventually dominate the pathogen population and the sensitive strain will be eliminated. Because of this, the high-risk fungicide will not affect the amount of disease and only the low-risk fungicide component of the mixture will be acting against disease. Hence, the high-risk fungicide becomes nonfunctional in the mixture and using the low-risk fungicide alone would have the same effect at a lower financial and environmental cost.

The work of Billard et al. (2011) provides a good example of a moderate fitness cost in the grapevine pathogen $B$. cinerea. Three isogenic lines were created carrying different mutations in the gene encoding the 3-ketoreductase target site of fenhexamid. All three mutant lines produced $\sim 20 \%$ fewer spores than the wild-type sensitive strain, indicating a fitness cost of $\sim 20 \%$. This is because the rate of spore production is a multiplicative factor in the compound transmission rate (Hobbelen et al. 2011), which is a reasonable measure of fitness in this context. If we apply this finding to Fig. 3, by drawing a vertical line up from the 0.2 cost on the $X$-axis (see dashed vertical line in Fig. 3), we can show that if the fungicides reduces the transmission rate by $70 \%$ (i.e., has $70 \%$ maximum efficacy, $k_{\mathrm{k}}=0.7$ ), a mixture can contain up to $\sim 54 \%$ of the high-risk fungicide without emergence of resistance. (This value is obtained from the intersection of the left dashed vertical line with the dotted curve in Fig. 3). If the fungicide has $50 \%$ efficacy, then a mixture can contain up to $\sim 74 \%$ of the high risk fungicide (similarly, the value is obtained from the intersection of the left dashed vertical line with the solid curve in Fig. 3). Finally, if the fungicide has $20 \%$ efficacy, then the mixture can contain up to $100 \%$ of the high-risk fungicide.

Resistance to the DMI flutriafol in the sugarbeet pathogen $C$. beticola presents an example of a high fitness cost (Karoglanidis et al. 2001). In this case, average spore production was $\sim 40 \%$ lower in nine strains resistant to flutriafol compared to 12 sensitive strains, indicating an average fitness cost of $\sim 40 \%$. We apply this finding to Fig. 3 by drawing a vertical line up from the 0.4 cost on the $X$-axis and find that a fungicide with $70 \%$ efficacy can contain up to $85 \%$ of the high-risk fungicide. With an efficacy of $50 \%$, the mixture can contain up to $\sim 98 \%$ of the high-risk fungicide and with an efficacy of $20 \%$, the mixture can have up to $100 \%$ high-risk fungicide without emergence of resistance.

For each of these examples, we assumed full resistance to the fungicide. If resistance is only partial, then more of the high-risk fungicide can be used without selecting for the emergence of resistance. We also assumed that the fitness cost was a single, constant value for all strains. If the fitness cost exhibits a distribution ranging across higher or lower values than the average shown in our example, then the calculation should be based upon the lowest measured fitness cost in order to avoid selecting for resistant strains. 
In the examples discussed above we used measurements of fitness cost to predict the optimal proportion of the two fungicides in the mixture theoretically, This prediction needs to be tested using field experiments, in which the amount of disease and the frequency of resistance are measured over time at different proportions of the high- and low-risk fungicides in the mixture. From these measurements the optimal proportions of the fungicides can be obtained empirically. It is this empirically determined optimal proportion of fungicides that is likely to be most useful for growers and fungicide producers to guide decisions about mixture strategies for managing fungicide resistance. Moreover, by comparing the optimal proportions obtained theoretically and empirically, one can evaluate the performance of a mathematical model and identify aspects of the model that need improvement.

\subsection{Fungicide mixture versus alternation}

As we discussed in Sec. 2 (also discussed in Shaw MW 2006) fungicide alternation can be effective in the presence of a fitness cost because selection for resistance may be reversed and resistant strains may be eliminated from the population. When using an alternation strategy, the period of selection during which the resistant strain is favored in the presence of the high-risk fungicide is followed by a period during which selection favors the sensitive strain in the absence of this fungicide. The latter period is typically much longer because the selection pressure induced by the high-risk fungicide is much larger than that induced by the fitness cost of resistance. Hence, one needs to wait for quite a long time before the resistant strain disappears and the high-risk fungicide can be used again. Moreover, there are times during which the frequency of the resistant strain becomes high (at the end of the period of the application of the high-risk fungicide), which increases the risk that resistance will spread to other regions. Both of these disadvantages are avoided by using a mixture where the proportion of the low-risk fungicide is above a critical value determined here (Fig. 3 ). In this case there is no need to delay the application of the high-risk fungicide and the frequency of the resistant strain does not rise above the mutation- or migration-selection equilibrium because the mixture does not induce selection for resistance.

\subsection{Dynamic turnover of fungicide mixtures}

In the presence of fitness costs, key advantages of both mixtures and alternations discussed above can be combined using the concept of dynamic turnover. Dynamic turnover means that the fungicides applied will need to change at regular intervals (eg within a growing season or between growing seasons) over time and space. It is likely that both mixtures and alternations of fungicides will also fail after prolonged use of the same mixture or alternation, but dynamic turnover of the components in the mixtures will be more likely to provide longer-term solutions. Hence, this approach will be an important aspect of any strategy that leads to durable disease control. The goal of dynamic turnover is to create a diverse chemical environment that imposes disruptive selection on the pathogen population, avoiding the consequences of constant directional selection that favors a particular set of resistance mutations. Dynamic turnover of fungicides presents the pathogen with the evolutionary dilemma of needing to put together a combination of mutations that provides resistance to a diverse set of chemical environments (Mikaberidze et al. 2014b).

\section{Conclusions}

Knowledge of fitness costs is crucial for predicting effectiveness and optimizing disease control strategies based on fungicide combinations, including mixtures, alternations and 
dynamic turnover of mixtures. We suggest that dynamic turnover of fungicide mixtures would be a superior strategy in terms of delaying the emergence of fungicide resistance. However, in order to determine optimal parameters for these strategies more field experimentation as well as additional modeling studies will be necessary. In particular, for polycyclic pathogens, fitness costs of resistant populations should be measured by taking the apparent growth rate, $r$, as a measure of fitness, because it is most relevant from the point of view of disease control. To the best of our knowledge, the fitness costs of fungicide resistant strains were not yet measured with respect to $r$. In the studies cited in Sec. 2 different components of fitness were measured that may or may not be related to $r$. Therefore, we identified a major gap in our knowledge of fitness costs. We hope this chapter will stimulate further experimental investigations to better characterize fitness costs.

\section{References.}

Anderson JB (2005) Evolution of antifungal-drug resistance: mechanisms and pathogen fitness. Nature reviews Microbiology 3:547-556

Billard A, Fillinger S, Leroux P, Lachaise H, Beffa R, Debieu D (2012) Strong resistance to the fungicide fenhexamid entails a fitness cost in Botrytis cinerea, as shown by comparisons of isogenic strains. Pest Management Science 68:684-691

Brent KJ, Hollomon DW (2007) Fungicide resistance: the assessment of risk. (2nd ed.) CropLife International, Belgium, pp 1-52

Chapara V, Taylor RJ, Pasche JS, Gudmestad NC (2011) Competitive parasitic fitness of mefenoxam-sensitive and -resistant isolates of Phytophthora erythroseptica under fungicide selection pressure. Plant Dis 95:691-696

Cools HJ (2008) Are azole fungicides losing ground against Septoria wheat disease? Resistance mechanisms in Mycosphaerella graminicola. Pest Manag Sci 64:681-684

Corio-Costet M-F, Dufour M-C, Cigna J, Abadie P, Chen W-J (2010) Diversity and fitness of Plasmopara viticola isolates resistant to QoI fungicides. Eur J Plant Pathol 129:315-329

Hobbelen PHF, Paveley ND, Fraaije B, Lucas J, van den Bosch F (2011) Derivation and testing of a model to predict selection for fungicide resistance. Plant Pathol 60:304-313

Holmes G, Eckert J (1995) Relative fitness of imazalil-resistant and -sensitive biotypes of Penicillium digitatum. Plant Dis 79:1068-1073

Hu JH, Hong CX, Stromberg EL, Moorman GW (2008) Mefenoxam sensitivity and fitness analysis of Phytophthora nicotianae isolates from nurseries in Virginia, USA. Plant Pathol 57:728-736

Iacomi-Vasilescu B, Bataille-Simoneau N, Campion C, Dongo A, Laurent E, Serandat I, Hamon B, Simoneau P (2008) Effect of null mutations in the AbNIK1 gene on saprophytic and parasitic fitness of Alternaria brassicicola isolates highly resistant to dicarboximide fungicides. Plant Pathol 57:937-947

Kadish D, Cohen Y (1992) Overseasoning of metalaxyl-sensitive and metalaxyl-resistant isolates of Phytophthora infestans in potato tubers weeks after inoculation. Phytopathology 82:887-889

Karaoglanidis G, Thanassoulopoulos CC, Ioannidis PM (2001) Fitness of Cercospora beticola field isolates-resistant and-sensitive to demethylation inhibitor fungicides. Eur J Plant Pathol 107:337-347

Karaoglanidis G, Luo Y, Michailides T (2011) Competitive ability and fitness of Alternaria alternata isolates resistant to QoI fungicides. Plant Dis 95:178-182

Kim YK, Xiao CL (2011) Stability and fitness of pyraclostrobin- and boscalid-resistant phenotypes in field isolates of Botrytis cinerea from apple. Phytopathology 101:1385-1391

Ma B, Uddin W (2009) Fitness and competitive ability of an azoxystrobin-resistant G143A mutant of Magnaporthe oryzae from perennial ryegrass. Plant Dis 93:1044-1049 
Mikaberidze A, McDonald B, Bonhoeffer S (2014a) How to develop smarter host mixtures to control plant disease? Plant Pathol, in press, pre-print arXiv:14022788

Mikaberidze A, McDonald BA, Bonhoeffer S (2014b) Can high risk fungicides be used in mixtures without selecting for fungicide resistance? Phytopathology 104:324-331

McDonald BA (2014) Using dynamic diversity to achieve durable disease resistance in agricultural ecosystems. Tropic Plant Pathol 39:191-196

Mundt CC (2002) Use of multiline cultivars and cultivar mixtures for disease management. Annu Rev Phytopathol 40:381-410

Peever TL, Milgroom MG (1994) Lack of correlation between fitness and resistance to sterol biosynthesis-inhibiting fungicides in Pyrenophora teres. Phytopathology 84:515

Peever T, Milgroom M (1995) Fungicide resistance - lessons for herbicide resistance management? Weed Technol 9:840

Shaw MW (2006) Is there such a thing as a fungicide resistance strategy? A modeller's perspective. Aspects Appl Biol 78:37-43

Stukenbrock EH, McDonald BA (2008) The origins of plant pathogens in agro-ecosystems. Annu Rev Phytopathol 46:75-100

Suzuki F, Yamaguchi J, Koba A (2010) Changes in fungicide resistance frequency and population structure of Pyricularia oryzae after discontinuance of MBI-D fungicides. Plant Dis 94:329-334

Torriani SF, Brunner PC, McDonald BA, Sierotzki H (2009) QoI resistance emerged independently at least 4 times in European populations of Mycosphaerella graminicola. Pest Manag Sci 65:155-162

Webber J (1988) Effect of MBC fungicide tolerance on the fitness of Ophiostoma ulmi. Plant Pathol 37:217-224

Zhan J, Stefanato F, McDonald BA (2006) Selection for increased cyproconazole tolerance in Mycosphaerella graminicola through local adaptation and in response to host. Mol Plant Pathol 7:259-268

Zhan J, McDonald BA (2013) Field-based experimental evolution of three cereal pathogens using a mark-release-recapture strategy. Plant Pathol 62:106-114 


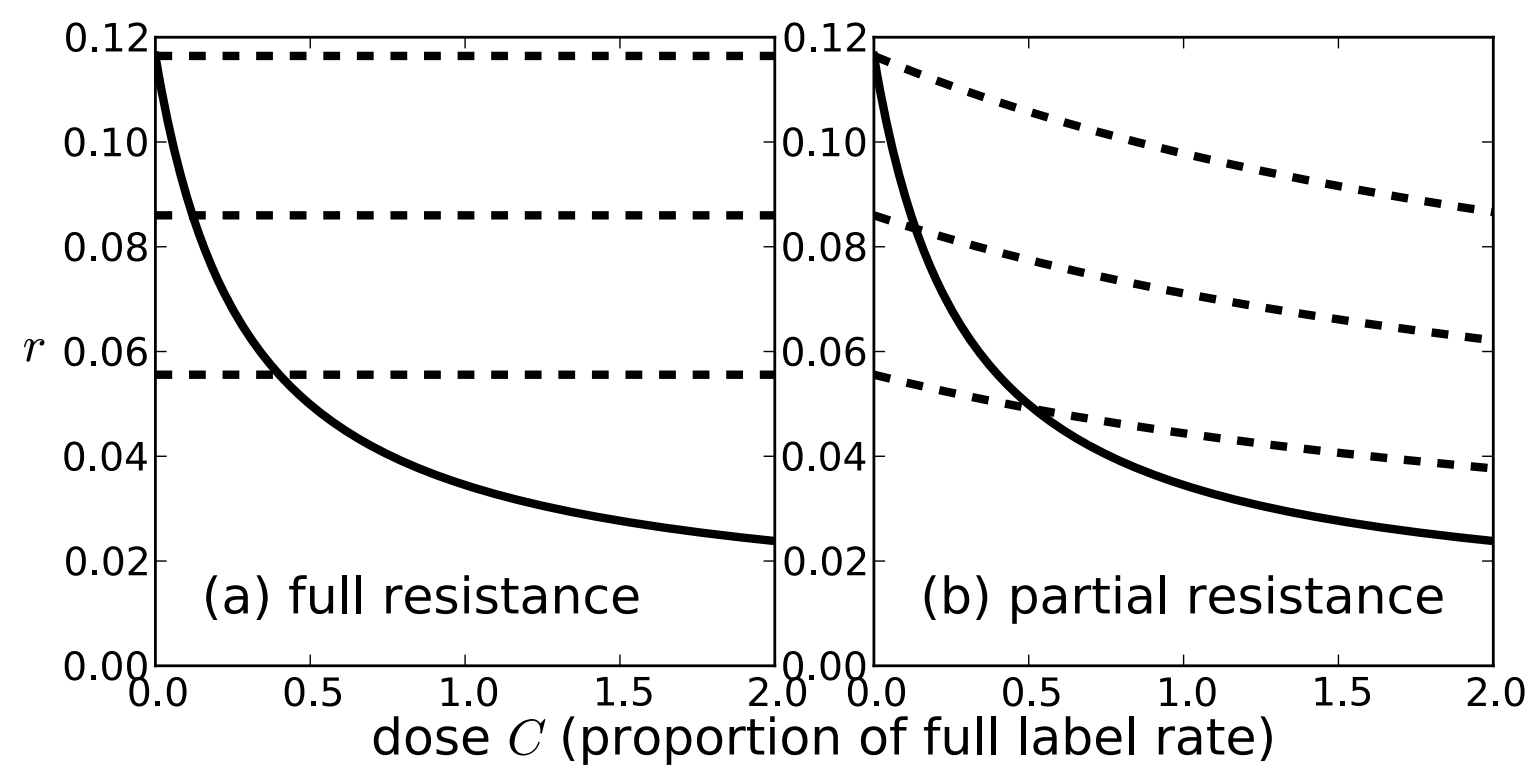

Fig. 1. The effect of fitness cost on selection for fungicide resistance. Apparent growth rate $r$ is plotted as a function of the fungicide dose $C$ for the sensitive pathogen strain [solid curve] and for the resistant pathogen strain [dashed curves] with the fitness cost $\rho_{r}=0$ (upper curve), $\rho_{r}=0.2$ (middle curve) and $\rho_{r}=0.4$ (lower curve). Other parameter values are: sensitive pathogen's life history traits, $r(C=0)=0.1173, R_{0}=4.26$, infectious period $1 / \mu=28$ days; dose-response parameters of the sensitive strain, $k_{\mathrm{k}}=0.7, D_{50}=0.3$; fungicide sensitivities of the resistant strain $\alpha_{1}=0.1, \alpha_{2}=0.7$. Parameter values are in the relevant range for Zymoseptoria tritici, an important pathogen of wheat.

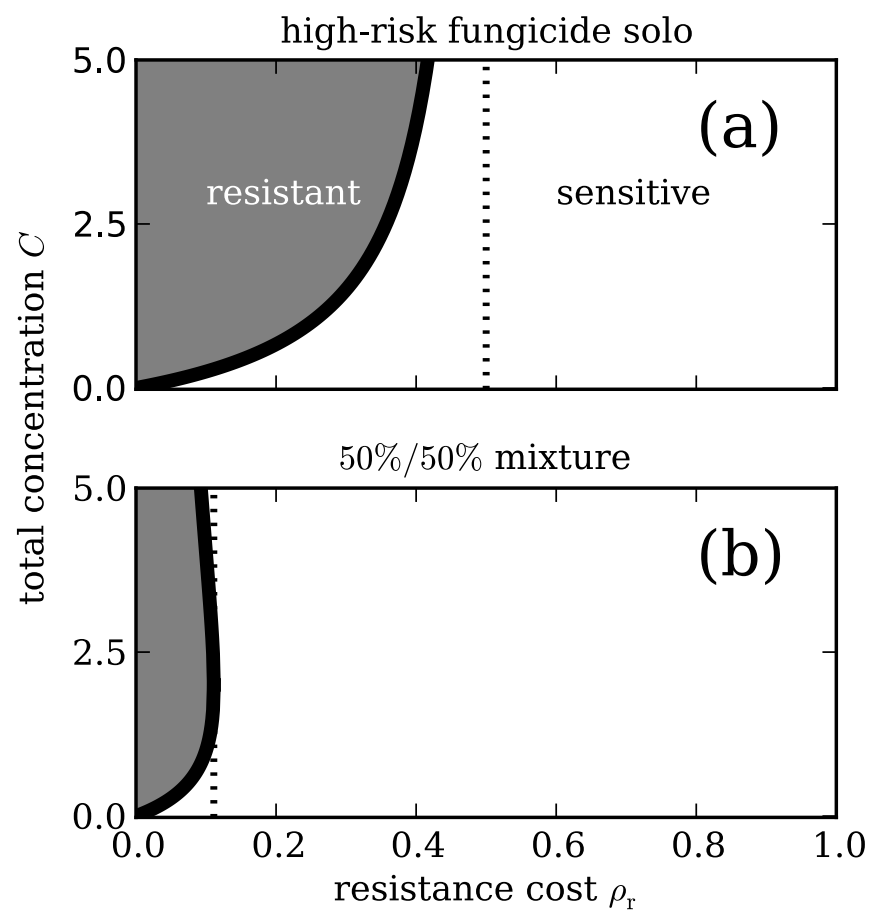

Fig. 2: Outcomes of the competition between the sensitive and resistant pathogen strains depending on the fitness cost of resistance $\rho_{\mathrm{r}}$ and the fungicide concentration $C$ when treated with a single fungicide $\mathrm{A}\left(C_{\mathrm{A}}=C\right.$, panel (a)) and the combination of fungicides $\mathrm{A}$ 
and $\mathrm{B}\left(C_{\mathrm{A}}=C_{\mathrm{B}}=C / 2\right.$, panel (b)). The range of the total fungicide concentration $C$ and the fitness cost of resistance $\rho_{\mathrm{r}}$, in which resistant strain is favored is shown in grey. The range where selection favors the sensitive strain is shown in white. Fungicides are assumed to have zero interaction $(u=0)$ and the resistant strain is assumed to be fully protected from fungicide $\mathrm{A}(\alpha=0)$, the fungicide dose-response parameters are $k_{\mathrm{k}}=0.6, C_{50}=1$. (see Sec. 3 for more details)

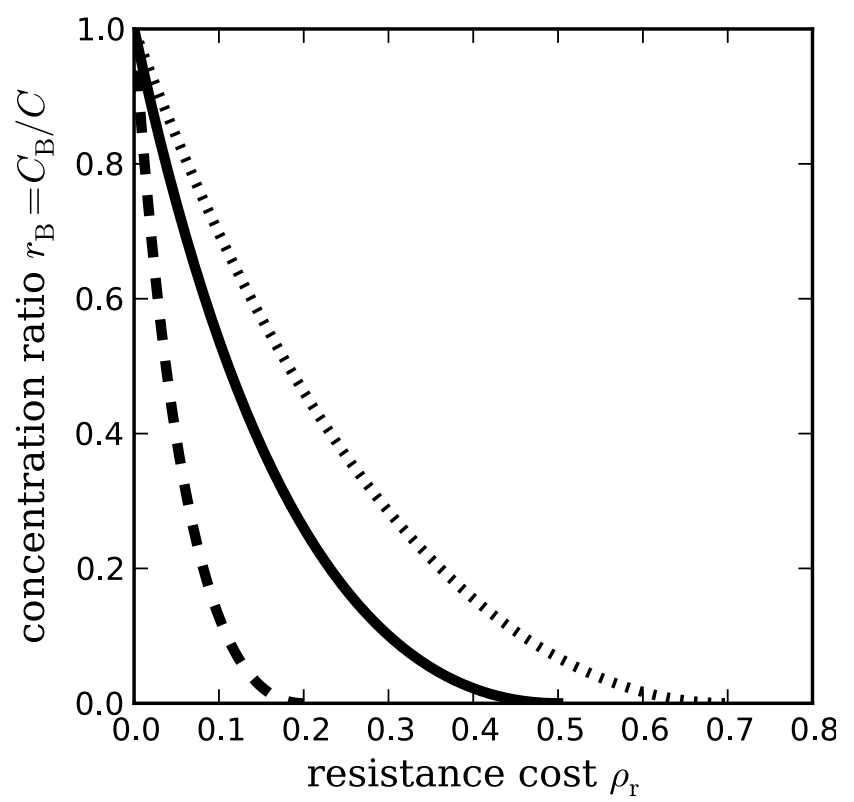

Fig. 3: The critical proportion $r_{\mathrm{Bc}}$ of fungicide $\mathrm{B}$ (low-risk fungicide) in the mixture, above which there is no selection for the resistant strain at any total fungicide dose $C$, plotted (black curve) and is a function of the resistance cost $\rho_{\mathrm{r}}$, assuming no pharmacological interaction ( $u=0)$, full resistance $(\alpha=0)$ and the maximum fungicide effect $k_{\mathrm{k}}=0.2$ (dashed), $k_{\mathrm{k}}=0.5$ (solid) and $k_{\mathrm{k}}=0.7$ (dotted). Vertical dashed lines indicate the estimates for the fitness costs $\rho_{r} \approx 0.2$ and $\rho_{r} \approx 0.4$ obtained from the studies (2001)Billard et al 2012 and (Karoglanidis et al 2001), correspondingly (see Sec. 4.1 for more details). 